\title{
Similar efectividad entre hipérico y paroxetina en el tratamiento de la depresión severa
}

Szegedi A, y col. BMJ. Mar 2005; 330:503.

\section{Objetivo}

Comparar la eficacia entre paroxetina e hipérico en extracto seco para el tratamiento de pacientes con depresión moderada a severa.

\section{Diseño}

Ensayo clínico, controlado, aleatorizado, doble ciego y multicéntrico.

\section{Lugar}

21 centros de atención psiquiatrica de Alemania

\section{Intervención}

Se incluyeron 251 pacientes adultos con depresión mayor con un puntaje mayor a 21 en la escala de depresión de Hamilton. La dosis inicial de hipérico fue $900 \mathrm{mg}$ en tres dosis diarias más un comprimido de placebo; mientras que la dosis inicial de paroxetina fue $20 \mathrm{mg}$ en un comprimido, más tres de placebo por día. Estas dosis iniciales se mantuvieron durante seis semanas, salvo en las personas que no habían respondido a las dos semanas, en quienes se incrementó la dosis a $1800 \mathrm{mg} /$ día de hipérico y a $40 \mathrm{mg} / \mathrm{día}$ de paroxetina.

\section{Medición de Resultados Principales}

El análisis se hizo por intención de tratar y el resultado principal se midió por los cambios en las escalas de depresión desde el valor

\section{basal hasta el del día 42}

Resultados principales

La valoración en la escala de depresión de Hamilton disminuyó en el grupo hipérico un promedio de 14 puntos y en el grupo paroxetina, un promedio de 11,4 . Ver tabla 1.

Tabla 1. Diferencia entre Hypericum y Paroxetina en la reducción al día 42 de los puntajes respecto de los basales en las distintas escalas de depresión e incidencia de efectos adversos.

\begin{tabular}{|c|c|c|c|c|}
\hline & Bppericum & Paroxetina & Diferencia e \\
\hline & & $n=122$ & $n=122$ & TCS5\% \\
\hline \multirow{3}{*}{$\begin{array}{l}\text { Puntaje promedio y } \\
\text { desvío estándar }\end{array}$} & Hamilton & $14,4(8,8)$ & $11.4(8,6)$ & $3,0(1,5-5,8)$ \\
\hline & Inventario de Beck & $16,6(10,7)$ & $12,6(10,6)$ & $3,0(1,4-6,5)$ \\
\hline & Montgomery-Asberg & $10,2(10,3)$ & $7,0(9,3)$ & $3,2(0,7-6,7)$ \\
\hline \multicolumn{2}{|l|}{ Incidencia de efect } & 0,035 & 0,06 & \\
\hline
\end{tabular}

\section{Conclusiones}

En el tratamiento de la depresión moderada a severa, el extracto de Hipérico perforatum es por lo menos tan efectivo y mejor tolerado que la Paroxetina.

Fuente de financiamiento: Dr. Willmar Schwabe Pharmaceuticals, manufacturer of WS 5570 .

\section{Comentario}

Si bien su eficacia ya había sido demostrada para el tratamiento de los síntomas depresivos leves a moderados en comparación con placebo, fluoxetina e imipramina ${ }^{1,2}$, este es el primer trabajo que incorpora Hipericum perforatum al ruedo de la investigación en el tratamiento de la depresión severa. Hasta ahora, el único estudio que comparaba hipérico con un antidepresivo sintético carecía del poder necesario para demostrar la bioequivalencia (no diferencia) ${ }^{3}$. El estudio es muy prolijo metodológicamente y pese a estar dirigido a demostrar bioequivalencia, el Hipérico mostró mayores beneficios, y menor reporte de efectos adversos. Este último dato es particularmente importante dado el riesgo de cronicidad de la depresión mayor y las nuevas evidencias que muestran los beneficios de tratamientos cada vez más prolongados. La comparación con paroxetina es apropiada ya que se trata de una droga utilizada habitualmente en ensayos de investigación y en la práctica clínica. Un indicador que muestra con claridad el efecto farmacológico del hipérico sobre la depresión es que en aquellos sujetos que inicialmente no respondían al tratamiento, el aumento de la dosis de ambas drogas produjo una disminución significativa en los puntajes de depresión comparable con el de los sujetos que respondieron inicialmente a dosis mas bajas. De todas maneras el conflicto de interés está presente aquí también, así como en otros tantos estudios financiados por la industria farmacéutica. El hipérico esta presente en la farmacopea local y se lo puede conseguir tanto en comprimidos de $300 \mathrm{mg}$ como en tinturas hidro-alcohólicas. Según la indicación de prescripción deberíamos indicar tres comprimidos diarios, o bien en forma de tintura madre al 30\% (30 gotas tres veces al día). Si bien existen prejuicios en la comunidad médica ante las medicaciones de origen natural o nó sintéticas, recordamos que muchos productos de uso corriente hoy en día provienen de la "naturaleza": Hioscina (antiespasmodico ampliamente usado de origen herbal) del Hiosciamus niger,Taxol (quimioterapico ahora de síntesis, obtenido de la corteza de un árbol)

Vincristina y Vinblastina (quimioterapicos alcaloides de la Vinca rosea) Enalapril (derivado de sintesis del "pril" original que se aisló del veneno de serpiente) estatinas (producto de síntesis cuyo precursor natural fue aislado de un hongo que contamina el arroz) Escopolamina, Digoxina, Osetalmivir, etc.

En cuanto al ensayo que comentamos, una de las limitaciones a tener en cuenta es que si bien muestra que el hipérico es igual y probablemente mejor que la paroxetina, su utilización está limitada por la gran cantidad de interacciones farmacológicas que posee ${ }^{4}$.

\section{Conclusiones del comentador}

El hipérico ha demostrado ser por lo menos igual a la paroxetina en el tratamiento de la depresión mayor y con menores efectos adversos. Para generalizar su utilización hace falta más tiempo de seguimiento, así como estudios de bioseguridad y vigilancia farmacológica; ya que dado su origen natural, aun faltan regulaciones y controles similares a las que se realizan respecto de las drogas sintéticas ${ }^{5}$.

Marcelo Ikonikoff [ Unidad de Medicina Familiar y Preventiva. Hospital Italiano de Buenos Aires. ]

Ikonikoff M. Similar efectividad entre hipérico y paroxetina en el tratamiento de la depresión severa. Evid. actual práct. ambul. 9(3):72. May-Jun. 2006. Comentado de: Szegedi A, Kohnen R, Dienel A. Acute treatmentof moderate to severe depression with hypericum extract WS 5570 (St John's wort): randomised controlled double blind non-inferority trial versus paroxetine. BMJ. Mar 2005; 330:503. PMID 15708844.

\section{Referencias}

1. Linde K, Munrow CD. St Johns Wort's for depression Cochrane Database Syst Rev 2004;(4):CD00048.

2. Helmut Woelk for the Remotiv/Imipramine Study Group. Comparison of St John's wort and imipramine for treating depression: randomised controlled trial. BMJ 2000;321:536-9

3. Vorbach EU, Hubner WD. Arnoldt KH et al. Efectiveness and tolerance of the hypericum extract in comparison with imipramine: Randomized double blind study in 135 outpatients. J Geriatric Psychiatric Neurol 1994;7: s17-23.

4. Stephen Bazire. Psychotropic drug directory. (2003-2004) 313-315.

5. Fefner RE, Beard K. Regulating herbal medicines in the UK. BMJ 2005; 331:62-63. 